\title{
Recent Advances in Understanding Audiovestibular Loss of a Vascular Cause
}

\author{
Hyun-Ah Kim, ${ }^{\text {a,b }}$ Hyung Lee Lab $^{\mathrm{a}, \mathrm{b}}$ \\ ${ }^{a}$ Department of Neurology, Keimyung University School of Medicine, Daegu, Korea \\ ${ }^{b}$ Brain Research Institute, Keimyung University School of Medicine, Daegu, Korea
}

Acute audiovestibular loss is characterized by abrupt onset of prolonged (lasting days) vertigo and hearing loss. Acute ischemic stroke in the distribution of the anterior inferior cerebellar artery (AICA) is known to be the leading cause of acute audiovestibular loss. So far, eight subgroups of AICA territory infarction have been identified according to the patterns of audiovestibular dysfunctions, among which the most common pattern is the combined loss of auditory and vestibular functions. Unlike inner ear dysfunction of a viral cause, which can commonly present as an isolated vestibular (i.e., vestibular neuritis) or cochlear loss (i.e., sudden deafness), labyrinthine dysfunction of a vascular cause rarely results in isolated loss of vestibular or auditory function. As audiovestibular loss may precede the central symptoms or signs of an ischemic stroke in the posterior circulation, early diagnosis and proper management of audiovestiubular loss may provide a window to prevent the progression of infarction to larger areas of the posterior circulation. A clinician should consider the possibility that acute audiovestibular loss may herald impending AICA territory infarction, especially when patients have basilar artery occlusive disease close to the origin of the AICA on brain MRA. This review aims to highlight the recent advances in understanding audiovestibular loss of a vascular cause and to address its clinical significance.

Keywords Audiovestibular loss; Vertigo; Hearing loss; Stroke; Anterior inferior cerebellar artery; Prodrome

\author{
Correspondence: Hyung Lee \\ Department of Neurology, Keimyung \\ University School of Medicine, 56 \\ Dalseong-ro, Jung-gu, Daegu 41931, \\ Korea \\ Tel: +82-53-250-7835 \\ Fax: +82-53-250-7840 \\ E-mail: hlee@dsmc.or.kr
}

Received: July 16, 2016

Revised: August 31, 2016

Accepted: October 9, 2016

This work was supported by the National Research Foundation of Korea (NRF) Grant funded by the Korean Government (MSIP) (No. 2014R1A5A2010008).

The authors have no financial conflicts of interest.

\section{Introduction}

Acute audiovestibular loss is one of the most common neurotological dysfunctions in patients presenting with acute vertigo. It is characterized by acute onset of prolonged (lasting days) vertigo and hearing loss. The required signs for diagnosing audiovestibular loss are canal paresis (CP) to caloric stimulation and sensorineural hearing loss on pure tone audiogram. Many neurological or neurotological conditions including viral inflammations, vascular insults, trauma, hereditary or genetic causes, bacterial meningitis, connective tissue disorders, or metabolic derangements should be considered in the differential diagnosis of audiovestibular loss. Among the vascular causes, ischemic stroke in the territory of the anterior inferior cerebellar artery (AICA) is known to be the leading cause of acute audiovestibular loss. ${ }^{1,2}$ It is usually associated with other neurological symptoms or signs, but can rarely occur in isolation (i.e., labyrinthine infarction). The aim of this review is to highlight the recent advances in understanding audiovestibular loss of a vascular cause and to address its clinical significance.

\section{Vascular territory and mechanism of audiovestibular loss of a vascular cause}

The internal auditory artery (IAA), the sole artery for supplying the inner ear, usually originates from the AICA, which is always a 
branch of the basilar artery., ${ }^{2,3}$ In $80 \%$ of people, the IAA is a branch of the AICA and sometimes derived directly from the basilar artery. ${ }^{3}$ In 2-3\% of the cases, the IAA is a branch of the posterior inferior cerebellar artery (PICA), which can explain the acute audiovestibular loss associated with cerebellar infarction in the PICA territory. ${ }^{3.4}$ Because territorial strokes of the AICA have commonly been associated with basilar artery branch occlusive disease, ${ }^{5,6}$ audiovestibular loss is commonly caused by the thrombotic narrowing of the AICA, or the basilar artery at the orifice of the AICA. Rarely, small emboli originating from the dissection in the vertebral artery lodge in the IAA causing inner ear infarction. ${ }^{7.8}$

\section{Lesion site of stroke in audiovestibular loss}

In theory, injury to any structures conveying the ascending vestibular and auditory pathways including the inner ear, eighth nerve, and vestibular and cochlear nuclei in the brainstem can cause audiovestibular loss with acute vertigo and hearing loss. In audiovestibular loss due to stroke, however, the lesions are mostly found in the inner ear, which is always supplied by the IAA. ${ }^{1,2}$ Because the inner ear requires a high-energy metabolism and the IAA is an end artery with little collateral from the otic capsule, the inner ear is particularly vulnerable to ischemia. ${ }^{9-12}$ By contrast, the eighth nerve in the auditory canal, the cerebellopontine angle, and the root entry zone is known to have a rich network of anastomosing vessels from the dural mater, petrous bone, AICA, PICA, and vertebral arteries. ${ }^{13-15} \mathrm{~A}$ patient ${ }^{16}$ with isolated audiovestibular loss showed a small infarction restricted to the root entry zone of the eighth nerve in the brainstem, suggesting that the root entry zone of the eighth nerve can be considered as a candidate site responsible for isolated audiovestibular loss. Isolated vestibular nucleus infarction has been recently identified as a cause of isolated vertigo, ${ }^{17,18}$ but isolated audiovestibular loss due to a focal infarction restricted to the vestibular and cochlear nuclei in the brainstem has not yet been identified in the literature. Indeed, the cochlear nuclei receive a rich blood supply from multiple sources, including branches of the AICA and PICA. ${ }^{3}$

\section{Frequency of stroke in audiovestibular loss}

As mentioned above, many neurological or neurotological diseases including AICA territory ischemic stroke can cause audiovestibular loss. Unfortunately, the frequency of audiovestibular loss associated with stroke among a consecutive series of the pa- tients with audiovestibular loss of various causes has not yet been identified in the literature. Instead, in a previous study, ${ }_{1}^{19}$ focused on the patients with acute hearing loss, 1.2\% (4/333) of the patients had acute hearing loss associated with posterior circulation ischemic stroke involving the cerebellum or brainstem. Among four patients with acute hearing loss due to stroke, two patients were identified as having audiovestibular loss due to accompanied $\mathrm{CP}$ to caloric stimulation on the side of hearing loss. Thus, $0.6 \%(2 / 333)$ of the patients with acute hearing loss had audiovestibular loss associated with posterior circulation ischemic stroke. ${ }^{19}$ Further studies are required to assess the prevalence of stroke related events in patients with audiovestibular loss.

\section{Pattern of audiovestibular loss due to ischemic injury to the inner ear in AICA territory infarction}

Up to now, eight subgroups of AICA territory infarction have been identified according to the patterns of audiovestibular dysfunctions. ${ }^{10,20,21}$ Among them, the most common pattern of audiovestibular dysfunction is combined loss of auditory and vestibular functions that was observed in approximately 60\% (49 of 82) of the patients from the largest series of AICA infarction. ${ }^{10}$ Therefore, audiovestibular loss is now considered an important sign for the diagnosis of AICA territory infarction. ${ }^{1}{ }^{10,19}$ The next common pattern of audiovestibular dysfunction is the absence of auditory or vestibular dysfunction, which was observed in approximately $32 \%$ (26/82) of patients from the aforementioned series. ${ }^{10}$ In this condition, vertigo results from dysfunction of the central vestibular structures including the cerebellum and/or brainstem. Isolated auditory loss (i.e., acute hearing loss on pure tone audiogram without $\mathrm{CP}$ to caloric stimulation), isolated superior vestibular loss (i.e., $\mathrm{CP}$ to caloric stimulation without impaired cervical vestibular evoked myogenic potential response or hearing loss), or isolated inferior vestibular loss (abnormal cervical vestibular evoked myogenic potential response without $\mathrm{CP}$ or hearing loss) are less commonly found. ${ }^{10,20,21}$ Rarely, isolated audiovestibular loss is a sole manifestation of AICA territory infarction, in which a tiny acute infarct seen on brain MRIs is asymptomatic. ${ }^{22}$

\section{Audiovestibular loss in non-AICA territory posterior circulation ischemic stroke}

As mentioned earlier, in most cases, the labyrinthine artery originates from the AICA, but in a few cases it can originate from the PICA or directly from the basilar artery. ${ }^{3}$ A PICA origin of the labyrinthine artery is found in 3 out of 100 temporal bone dis- 
sections. ${ }^{13}$ A previous study showed that in a series of 685 consecutive cases of posterior circulation ischemic stroke diagnosed with brain MRIs, 5 patients $(0.7 \%)$ were identified as having audiovestibular loss from PICA territory cerebellar infarction. ${ }^{4} \mathrm{Au}$ diovestibular loss due to non-AICA territory posterior circulation infarction is commonly associated with cerebellar infarction in the territory of the PICA. Considering that the known variations of the anatomy of the IAA, and results of audiological evaluations, audiovestibular loss due to non-AICA territory posterior circulation infarct probably results from damage to the peripheral auditory system within the inner ear. ${ }^{4}$
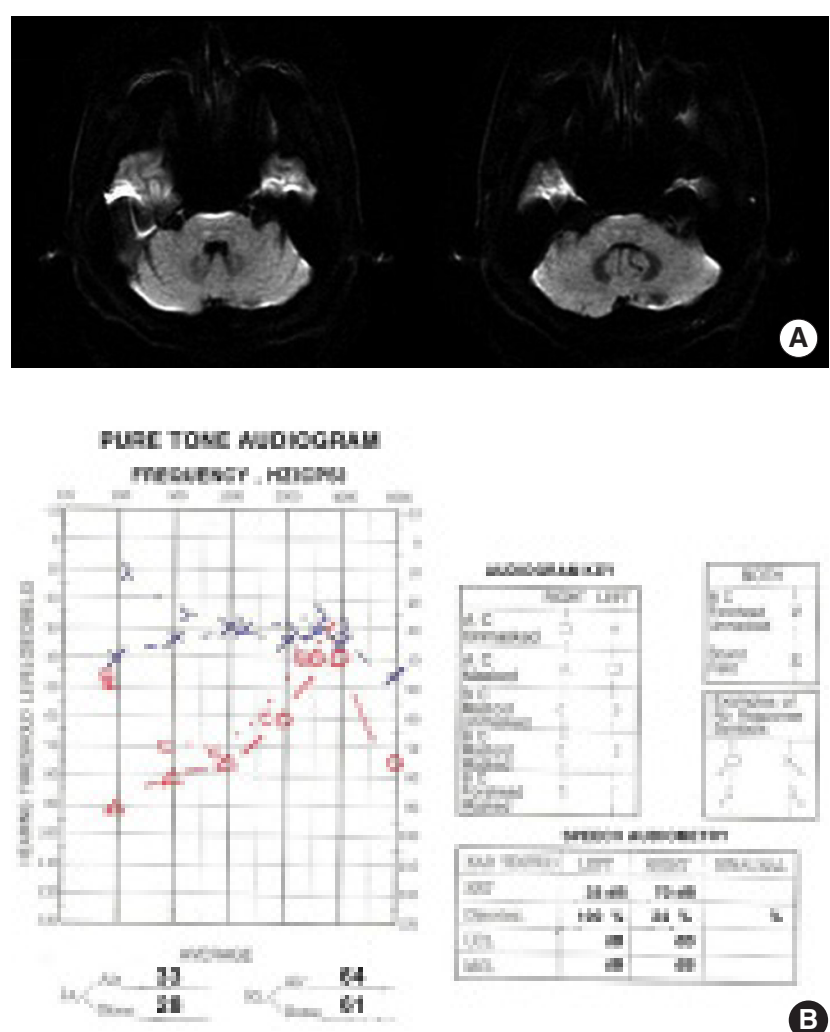

B

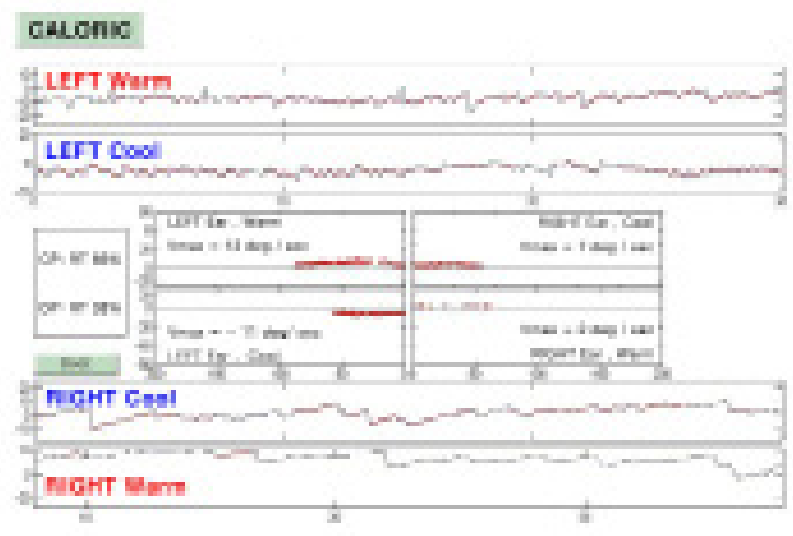

C

\section{Audiovestibular disturbance as a sole manifestation of transient ischemic attack}

There have been several case reports, ${ }_{1}^{23-25}$ indicating that acute audiovestibular disturbance with hearing loss and vertigo may precede the central symptoms or signs due to posterior circulation ischemic stroke (mainly in the distribution of the AICA). In the first study to investigate consecutive patients entered into a prospective acute registry, approximately 31\% (5/16) of the patients had an acute audiovestibular disturbance such as hearing
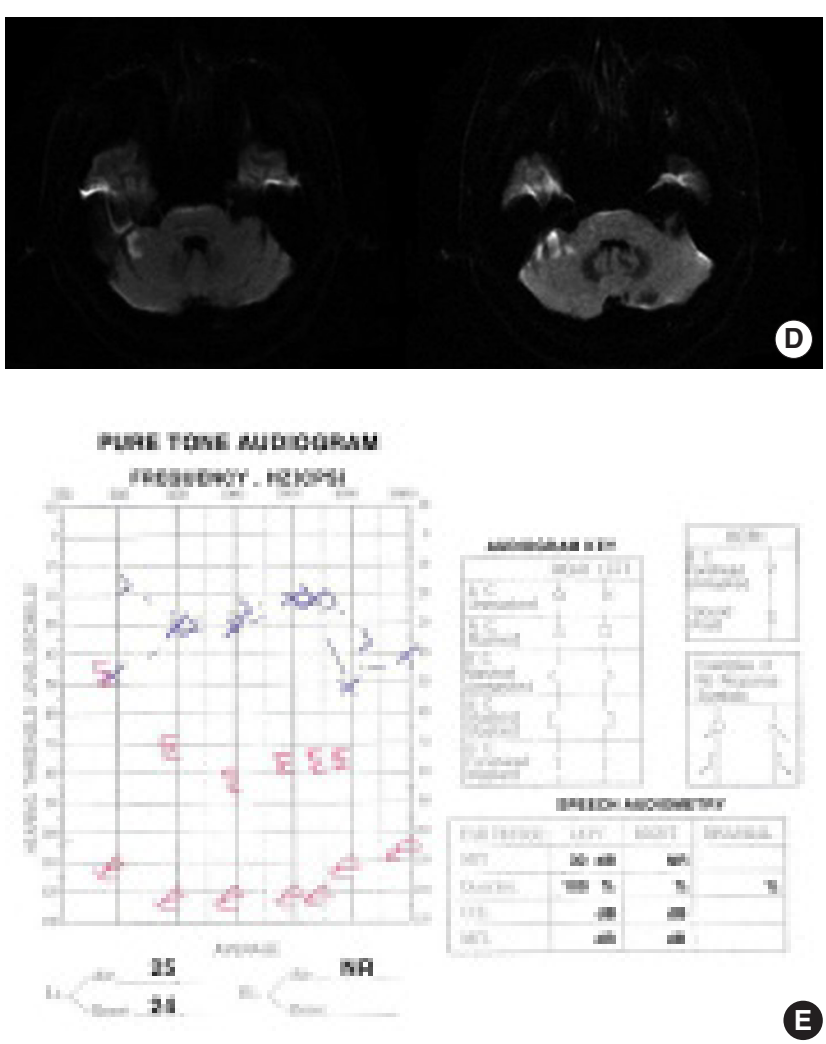

Figure 1. MRI and audiovestibular findings in a patient with recurrent vertigo and fluctuating hearing loss with tinnitus - prodromal signs of AICA territory infarction. (A) Normal axial diffusion-weighted brain MRI 1 day after the onset of recurrent vertigo and fluctuating rightsided hearing loss with tinnitus. (B) An initial pure tone audiogram performed reveals moderate degree of hearing loss with 24\% speech discrimination in the right ear. Hearing levels in decibels (dB) (American National Standards Institute, 1989) are plotted against stimulus frequency on a logarithmic scale. (C) Video-oculographic recordings of bithermal caloric tests performed showed right canal paresis (65\%). Three days later, patient complained of exacerbation of vertigo and hearing loss on the right ear. On examination, there was bidirectional gaze-evoked nystagmus and dysmetria on the right limb. (D) Follow-up axial diffusion-weighted MRI demonstrated hyperintense foci involving the right anterior cerebellum including the flocculus. (E) Follow-up pure tone audiogram shows aggravated profound hearing loss on the right ear. AICA, anterior inferior cerebellar artery; Vmax, maximal velocity of slow phase of nystagmus. 
Table 1. Frequency and characteristics of acute audiovestibular disturbance before AICA territory infarction

\begin{tabular}{|c|c|c|c|}
\hline & Lee and $\mathrm{Cho}^{15}$ & Kim et al. ${ }^{26}$ & Lee et al. ${ }^{10}$ \\
\hline Number of studied patients & 16 & 54 & 82 \\
\hline \multicolumn{4}{|l|}{ Pattern of AICA territory infarction } \\
\hline Isolated AICA & 12 & 36 & 55 \\
\hline AICA plus & 4 & 18 & 27 \\
\hline Percentage of acute audiovestibular disturbance before VBIS & $31 \%(5 / 16)$ & $8 \%(4 / 54)$ & $16 \%(13 / 82)$ \\
\hline Identification of normal MRI at the stage of isolated audiovestibular disturbance & None & Complete & Incomplete \\
\hline Identification of $\mathrm{CP}$ at the stage of isolated audiovestibular disturbance & None & Complete & Incomplete \\
\hline Identification of SNHL on PTA at the stage of isolated audiovestibular disturbance & None & Complete & Incomplete \\
\hline \multicolumn{4}{|l|}{ Pattern of audiovestibular disturbance before VBIS } \\
\hline Vertigo/hearing loss/tinnitus & 3 & 3 & 3 \\
\hline Vertigo/tinnitus & 1 & 0 & 1 \\
\hline Vertigo/Hearing & 1 & 1 & 9 \\
\hline Interval from audiovestibular disturbance to VBIS & 10 days & 4 days & 30 days \\
\hline \multicolumn{4}{|l|}{ Kinetics of audiovestibular disturbance } \\
\hline Plus other symptoms & 0 & 0 & 0 \\
\hline Isolated & 5 & 4 & 13 \\
\hline
\end{tabular}

$\mathrm{CP}$, canal paresis; AICA, anterior inferior cerebellar artery; SNHL, sensorineural hearing loss; PTA, pure tone audiogram; VBIS, vertebrobasilar ischemic stroke.

loss, vertigo and/or tinnitus as a prodrome of AICA territory infarction 1 to 10 days before the onset of other brainstem or cerebellar symptoms. ${ }^{15}$ In another study, follow-up MRIs of patients who first presented with isolated audiovestibular loss found that 8\% (4/43) of the patients with documented AICA territory infarction had normal brain MRIs on initial presentation. ${ }^{26}$ This study was the first to document the audiovestibular loss using caloric test and pure tone audiogram at the time of isolated vertigo and hearing loss. In a more recent study, ${ }_{1}^{10}$ approximately $16 \%(13 / 82)$ of the patients with AICA territory infarction on brain MRIs had an episode(s) of transient vertigo, hearing loss and/or tinnitus within a month before the infarction. All of these data suggested that $8-31 \%$ of patients with AICA territory infarction have acute audiovestibular disturbance before the onset of other central symptoms or signs from more widespread infarction. Figure 1 illustrates the MRIs and audiovestibular findings in a patient with acute audiovestibular loss as a prodromal sign of AICA territory infarction. The diffusion-weighted MRI was normal at the time of acute isolated audiovestibular loss. The frequency and pattern of audiovestibular disturbance before AICA territory infarction are summarized in Table 1.

\section{What factor(s) suggests impending stroke at the stage of isolated audiovestibular disturbance?}

If audiovestibular disturbance with vertigo, hearing loss, and/ or tinnitus occurs before the potentially more disabling symptoms and signs suggesting widespread posterior circulation ischemic stroke, its recognition may provide a window of opportunity for intervention in the hope of limiting long-term disability. ${ }^{21} \mathrm{Al}-$ though there are as yet no systematic data on which factor suggests impending posterior circulation ischemic stroke or which intervention might be beneficial at the stage of isolated audiovestibular loss, patients with prodromal audiovestibular disturbance were more likely to have focal or diffuse stenosis of the basilar artery close to the origin of the AICA than patients without audiovestibular disturbance. ${ }^{10,15}$ This finding suggests that AICA infarction should be considered in patients with vascular risk factors and acute audiovestibular loss, especially when brain MRA showed stenosis of the basilar artery close to the origin of the AICA, even when MRls does not demonstrate acute infarction in the brain. 10,15,21,27 Overall, because acute audiovestibular disturbance can be the harbinger of more widespread posterior circulation ischemic stroke, early recognition of the implications for proper investigation and treatment appears to have taken on a new urgency. ${ }^{21,26,28,29}$

\section{Can a vascular cause serve a common mechanism of isolated vertigo (i.e., vestibular neuritis) or isolated hearing loss (i..., sudden deafness)?}

In a case of isolated vertigo in elderly patients with risk factors for stroke, the superior vestibular labyrinth is a possible candidatesite for isolated vertigo because anterior vestibular artery (AVA), the sole artery for supplying the superior vestibular labyrinth is small and has little collateral circulation. ${ }^{9-12} \mathrm{~A}$ previous report ${ }^{11}$ also supported this assumption because approximately $50 \%$ of patients with isolated episodic vertigo of a vascular 
cause (i.e., vertebrobasilar insufficiency) had unilateral $\mathrm{CP}$, which is commonly ascribed to damage to the inner ear (i.e., superior vestibular labyrinth). However, a recent study reported a contradictive finding in that only four $(4 / 82,5 \%)$ patients showed isolated vestibular labyrinthine involvement at the time of AICA infarction. ${ }^{10}$ Thus, although isolated AVA infarction may serve a mechanism of isolated vascular vertigo, the incidence would be low. Indeed, ischemic injury to the inner ear rarely results in isolated loss of vestibular or cochlear function, whereas the viral inflammatory injury commonly presents an isolated vestibular (i.e., vestibular neuritis) or cochlear loss (i.e., sudden deafness). ${ }^{10,} 21$ Clinicians should be aware of the possibility of a vascular cause when combined audiovestibular loss occurs in elderly patients presenting with prolonged vertigo. ${ }^{10,21}$

\section{How can we differentiate acute labyrinthine infarction from acute viral labyrinthitis?}

At present, the differentiation of acute labyrinthine infarction from acute labyrinthitis mostly depends on careful history and detailed examination. ${ }^{30-33}$ History is the key cornerstone in differentiating these two conditions. For example, for a young patient who had a history of viral infection such as, upper respiratory infection, before the onset of audiovestibular loss, a viral cause is the most likely considered. By contrast, the presence of vascular risk factors in elderly patients may suggest a vascular cause. Neurological examination may also help to differentiate these two conditions. When audiovestibular loss has occurred as a component of a larger neurological syndrome with brainstem or cerebellar signs, audiovestibular loss of a vascular cause (i.e., labyrinthine infarction) is highly suggested.

To assess for a vascular cause in patients with transient vertigo, a recent study ${ }^{34}$ suggested that the use of $A B C D^{2}$ score, ${ }^{35}$ a clinical prediction tool to assess the risk of stroke after a transient ischemic attack, may predict cerebrovascular attacks in patients with transient vertigo. However, the diagnostic utility of the $A B C D^{2}$ score as a clinical tool for differentiating the causes of acute onset of isolated prolonged (not transient) vertigo and hearing loss has not been investigated. We need a further research to assess the clinical usefulness of the $A B C D^{2}$ score for differentiating a vascular cause from a viral cause in patients with audiovestibular loss. Overall, without pathological confirmation, it is impossible to precisely determine the cause of acute audiovestibular loss. Thus, clinicians should pay careful attention to the clinical signs and symptoms to differentiate audiovestibular loss of a vascular cause from acute labyrinthitis.. ${ }^{29,31}$ We need a further research to determine the clinical predictors of isch- emic versus viral cause of audiovestibular loss and to develop confirmatory tests. ${ }^{27,31}$

\section{Conflict of interest}

Dr. Lee serves on the editorial boards of the Research in Vestibular Science, Frontiers in Neuro-otology, and Current Medical Imaging Review.

\section{References}

1. Lee $H$, Sohn SI, Jung DK, Cho YW, Lim JG, Yi SD, et al. Sudden deafness and anterior inferior cerebellar artery infarction. Stroke 2002;33:2807-2812.

2. Lee H. Neuro-otological aspects of cerebellar stroke syndrome. J Clin Neurol 2009;5:65-73.

3. Häusler R, Levine RA. Auditory dysfunction in stroke. Acta Otolaryngol 2000;120:689-703.

4. Lee H. Sudden deafness related to posterior circulation infarction in the territory of the nonanterior inferior cerebellar artery: frequency, origin, and vascular topographical pattern. Eur Neurol 2008;59:302-306.

5. Amarenco $P_{1}$ Hauw JJ. Cerebellar infarction in the territory of the anterior and inferior cerebellar artery. Brain 1990;113:139155.

6. Amarenco P, Rosengart A, Dewitt LD, Pessin MS, Caplan LR. Anterior inferior cerebellar artery territory infarcts. Mechanism and clinical features. Arch Neurol 1993;50:154-161.

7. Raupp SF, Jellema K, Sluzewski M, de Kort PL, Visser LH. Sudden unilateral deafness due to a right vertebral artery dissection. Neurology 2004;62:1442.

8. Choi KD, Chun JU, Han MG, Park SH, Kim JS. Embolic internal auditory artery infarction from vertebral artery dissection. J Neurol Sci 2006;246:169-167.

9. Oas JG, Baloh RW. Vertigo and the anterior inferior cerebellar artery syndrome. Neurology 1992;42:2274-2279.

10. Lee H, Kim JS, Chung EJ, Yi HA, Chung IS, Lee SR, et al. Infarction in the territory of anterior inferior cerebellar artery: spectrum of audiovestibular loss. Stroke 2009;40:3745-351.

11. Grad A, Baloh RW. Vertigo of vascular origin: clinical and electronystagmographic features in 84 cases. Arch Neurol 1989;46: 281-284.

12. Kim JS, Lee $H$. Inner ear dysfunction due to vertebrobasilar ischemic stroke. Semin Neurol 2009;29:534-540.

13. Mazzoni A. Internal auditory canal. Arterial relations at the porus acusticus. Ann Otol Rhinol Laryngol 1969;78:797-814.

14. Mazzoni A. Internal auditory artery supply to the petrous bone. Ann Otol Rhinol Laryngol 1972;81:13-21. 
15. Lee $\mathrm{H}$, Cho YW. Auditory disturbance as a prodrome of anterior inferior cerebellar artery infarction. J Neurol Neurosurg Psychiatry 2003;74:1644-1648.

16. Choi SY, Park JH, Kim HJ, Kim JS. Vestibulocochlear nerve infarction documented with diffusion-weighted MRI. J Neurol 2015;262:1363-1365.

17. Kim HA, Lee $\mathrm{H}$. Isolated vestibular nucleus infarction mimicking acute peripheral vestibulopathy. Stroke 2010;41:1558-1560.

18. Kim HJ, Lee SH, Park JH, Choi JY, Kim JS. Isolated vestibular nuclear infarction: report of two cases and review of the literature. J Neurol 2014;261:121-129.

19. Sauvaget $E_{1}$ Kici $S$, Petelle $B$, Kania $R$, Chabriat $H_{1}$ Herman $P_{\text {, et }}$ al. Vertebrobasilar occlusive disorders presenting as sudden sensorineural hearing loss. Laryngoscope 2004;114:327-332.

20. Ahn BH, Kim HA, Yi HA, Oh SY, Lee H. Abnormal cervical vestibular-evoked myogenic potential in anterior inferior cerebellar artery territory infarction: Frequency, pattern, and a determinant. J Neurol Sci 2011;307:114-119.

21. Lee $\mathrm{H}$. Audiovestibular loss in anterior inferior cerebellar artery territory infarction: a window to early detection? J Neurol Sci 2012;313:153-159.

22. Lee $H, A h n B H, B a l o h ~ R W$. Isolated sudden deafness with vertigo as a sole manifestation of anterior inferior cerebellar infarction. J Neurol Sci 2004;222:105-107.

23. Lee $H$, Whitman GT, Lim JG, Lee SD, Park YC. Bilateral sudden deafness as a prodrome of anterior inferior cerebellar artery infarction. Arch Neurol 2001;58:1287-1289.

24. Yi HA, Lee $H$, Lee $S R$, Ahn BH, Park BR, Whitman GT. Sudden deafness as a sign of stroke with normal diffusion-weighted brain MRI. Acta Otolaryngol 2005;125:1119-1121.
25. Lee H, Kim HJ, Koo JW, Kim JS. Progression of acute cochleovestibulopathy into anterior inferior cerebellar artery infarction. J Neurol Sci 2009;278:119-122.

26. Kim JS, Cho KH, Lee $\mathrm{H}$. Isolated labyrinthine infarction as a harbinger of anterior inferior cerebellar artery territory infarction with normal diffusion-weighted brain MRI. J Neurol Sci 2009;278:82-84.

27. Kim HA, Lee $S R$, Lee $H$. Acute peripheral vestibular syndrome of a vascular cause. J Neurol Sci 2007;254;99-101.

28. Mort DJ, Bronstein AM. Sudden deafness. Curr Opin Neurol 2006;19:1-3.

29. Choi KD, Lee $H$, Kim JS. Vertigo in brainstem and cerebellar strokes. Curr Opin Neurol 2013;26:90-95.

30. Lee H. Isolated vascular vertigo. J Stroke 2014;16:124-130.

31. Lee $H$. Recent advances in acute hearing loss due to posterior circulation ischemic stroke. J Neurol Sci 2014;338:23-29.

32. Kim HA, Lee $H$. Recent advances in central acute vestibular syndrome of a vascular cause. J Neurol Sci 2012;321:17-22.

33. Kim HA, Yi HA, Lee $H$. Recent advances in cerebellar ischemic stroke syndromes causing vertigo and hearing loss. Cerebellum 2016;15:781-788.

34. Navi BB, Kamel H, Shah MP, Grossman AW, Wong C, Poisson $\mathrm{SN}$, et al. Application of the ABCD2 score to identify cerebrovascular causes of dizziness in the emergency department. Stroke 2012:43:1484-1489.

35. Johnston SC, Rothwell PM, Nguyen-Huynh MN, Giles MF, Elkins JS, Bernstein $A L$, et al. Validation and refinement of scores to predict very early stroke risk after transient ischaemic attack. Lancet 2007;369:283-292. 